\title{
Evaluation of enhanced haemolysis agar for detection of Listeria spp. and L. monocytogenes from production lines of fresh to cold-smoked fish
}

\author{
Manuela Vaz-Velho a,b, *, Gabriela Duarte ${ }^{\mathrm{a}}$, Paul Gibbs ${ }^{\mathrm{a}, \mathrm{c}}$ \\ ${ }^{\text {a }}$ Escola Superior de Biotecnologia, Universidade Católica Portuguesa, Porto, Portugal \\ ${ }^{\mathrm{b}}$ Escola Superior de Tecnologia e Gestão, Instituto Politécnico, Viana do Castelo, Portugal \\ ${ }^{\mathrm{c}}$ Leatherhead Food Research Association, Surrey, England, UK
}

\begin{abstract}
Enhanced haemolysis agar (EHA) was compared to the two conventional Listeria isolation agars Oxford and PALCAM for its ability to detect Listeria spp. from production lines of fresh to cold-smoked fish. The ability of EHA for distinguishing L. monocytogenes colonies from other Listeria spp. was also evaluated.

A total of 243 fish and environmental samples were analysed. Overall, 42 samples were found to contain Listeria spp. Only 34 samples were positive simultaneously by the three plating media. Two samples considered to be negative by the two conventional agars were found to be positive after isolation on EHA. All three selective agars were shown to be less effective in recovering Listeria spp. after primary enrichment in half-Fraser broth, compared to secondary enrichment in Fraser broth after 24 and $48 \mathrm{~h}$.

From 79 Listeria but presumptive negative L. monocytogenes colonies, EHA identified correctly 76 Listeria spp. and presented three false-negative results three colonies further identified as L. monocytogenes but showing no noticeable haemolysis on EHA. Twenty-three of the thirty-three L. monocytogenes presumptive positive colonies, were confirmed positive and ten were identified as L. seeligeri.

Despite its ability of distinguishing L. monocytogenes from the other Listeria spp., unless it is produced as a commercial medium, EHA cannot be an alternative to time-consuming classical identification because the preparation of this medium is both time and labour intensive.
\end{abstract}

\section{Introduction}

Listeria monocytogenes is the only species of the genus Listeria that has been involved in known

\footnotetext{
* Corresponding author. Escola Superior de Biotecnologia, Rua Dr. António Bernardino de Almeida, 4200 Porto, Portugal. Tel.: +351-22-5580043; fax: +351-22-5090351.

E-mail address: manuela@esb.ucp.pt (M. Vaz-Velho).
}

foodborne outbreaks of listeriosis. Only the haemolytic species of Listeria-L. monocytogenes, L. ivanovii, L. seeligeri-are associated with human pathogenicity. L. ivanovii has been reported to be involved in human pathology only rarely, and $L$. seeligeri has been reported only once to be the cause of meningitis in a non-immuno-compromised adult (Lovett and Twedt, 1988). The pathogen has been consistently isolated from production lines of fresh 
to cold-smoked fish (Farber, 1991; Hartemink and Georgsson, 1991; Dillon et al., 1992; Gibson, 1992; Hudson et al., 1992; Ben Embarek, 1994; Fuchs and Nicolaides, 1994; Jemmi and Keusch, 1994; Duarte et al., 1995; Eklund et al., 1995; Rørvik et al., 1995; Loncarevic et al., 1996; Jørgensen and Huss, 1998; Vaz-Velho et al., 1998; Autio et al., 1999). A suspected outbreak of listeriosis caused by cold-smoked salmon-trout and involving nine people was recently reported (Ericsson et al., 1997).

The commercially available plating media for Listeria can mask the presence of $L$. monocytogenes, and, even the selection of five suspect colonies at random from such agar media could lead to the detection only of non-pathogenic Listeria, even though a few L. monocytogenes were present on the plate (Beumer et al., 1997). Furthermore, L. innocua has been reported to grow faster than L. monocytogenes in selective enrichment broths, which increases the difficulty of isolating this last species (MacDonald and Sutherland, 1994). Therefore, the introduction of an isolation medium on which the pathogen can be differentiated from the non-pathogenic Listeria spp., can be achieved using enhanced haemolysis agar (EHA; Beumer et al., 1997). On EHA, L. monocytogenes and L. seeligeri can lyse ovine erythrocytes in the presence of the enzyme sphingomyelinase. L. ivanovii does not show a noticeable haemolysis on EHA.

\section{Materials and methods}

A total of 243 samples were analysed. Forty-two environmental and 191 fish samples were collected, respectively, at sites before the production head and along the process line of three Portuguese coldsmoking fish plants. All the smoking plants use fresh salmon, imported from Norway, salmon trout from two Portuguese trout farms and swordfish from different suppliers. The fresh salmon from the importer and the salmon trout, water, containers, ice, and polystyrene boxes of the trout farms were also analysed. Ten samples of smoked salmon and swordfish included in this study were collected from a retail outlet.

The fresh salmon and salmon-trout samples and the environmental samples from the trout farms were transported to the laboratory inside cold portable insulated boxes, refrigerated overnight and analysed the day after.

Due to the distance between the fish smoking plants and the laboratory $(600 \mathrm{~km})$, all the environmental and fish samples along the smoking processing chain of each factory were maintained in refrigerated conditions and were analysed up to a week after collection.

\subsection{Sampling procedure}

Ten centimetres square of the fresh fish skin and surfaces were swabbed (five swabs per sample) and the swabs were placed in $25 \mathrm{ml}$ of $0.1 \%(\mathrm{w} / \mathrm{v})$ peptone water $(1 \mathrm{~g} / 1$ Tryptone (LabM, MC5, Bury, UK) $+5 \mathrm{~g} / 1 \mathrm{NaCl}$ (Merck, 6404, Darmstadt, Germany)). Water samples were collected in sterile 500$\mathrm{ml}$ bottles for further filtration. Twenty-five grams of processed fish samples was collected in sterile plastic bags. The samples were transported to the laboratory inside cold portable insulated boxes.

\subsection{Isolation procedure}

The ISO 11290-1 (1996) analytical protocol was followed. For the swabbed samples, a pre-enrichment step was introduced in the protocol- $-0.1 \%$ peptone water was chosen according to Eklund et al. (1995) sampling procedure for cold-smoked fish processing equipment surfaces. Furthermore, it has been concluded that this pre-enrichment step improves the recovery of $L$. monocytogenes in the same type of samples as well as in fresh fish skin (Vaz-Velho et al., 2001). The water and ice samples were filtered (0.45 $\mu \mathrm{m}, \varnothing$ 47-mm membrane filters, Gelman Sciences, MI, USA) and the filters were placed in $20 \mathrm{ml}$ of primary enrichment broth, half-Fraser broth (Merck, 110398.0500) that contains half of the concentration of the selective supplement (Merck, 110399.0001) compared to Fraser broth. Swabs of the fresh fish and environmental samples in $25 \mathrm{ml}$ of peptone water were transferred to $225 \mathrm{ml}$ of primary enrichment broth and mixed. The $25 \mathrm{~g}$ of processed fish was placed in $225 \mathrm{ml}$ of primary enrichment broth and homogenised in a stomacher (Seward 400) for $2 \mathrm{~min}$. All the samples were incubated at $30^{\circ} \mathrm{C}$ for $24 \mathrm{~h}$. Aliquots of primary enrichment cultures 
$(0.1 \mathrm{ml})$ were transferred to $10 \mathrm{ml}$ of secondary enrichment Fraser broth and incubated at $35^{\circ} \mathrm{C}, 24-$ $48 \mathrm{~h}$. Primary and secondary enrichment cultures, whether showing growth or not, were sub-cultured by streaking onto Oxford (Merck, 107004.0500), PALCAM (Merck, 11755.0500) and EHA (Beumer et al., 1997) selective media. The Oxford and PALCAM plates were incubated at $30^{\circ} \mathrm{C}$ for $48 \mathrm{~h}$ and the EHA plates at $37^{\circ} \mathrm{C}$ for $48 \mathrm{~h}$. EHA has the following composition: Trypticase soy blood agar base EH (Difco 0028-17-9) 40 g/1, 4-methylumbelliferyl- $\beta$ D-glucoside (MUßG, Sigma M3633) $50 \mathrm{mg} / 1, \mathrm{LiCl}$ 5g/1, PALCAM supplement (Merck, 12122.0001) two vials, sphingomyelinase (Sigma S8633) 10 units, and sheep blood (sterile, defibrinated) $50 \mathrm{ml}$. The medium was prepared following the protocol of Beumer et al. (1997). The EHA plates were observed under daylight and under UV light at $366 \mathrm{~nm}$. Typical Listeria colonies on EHA under UV light (366 $\mathrm{nm}$ ) are surrounded by a very diffuse zone of light, whitish-blue fluorescence, with a yellow matt fluorescent centre after $48 \mathrm{~h}$ of incubation. Under daylight, they are translucent, shiny, entire, whitish to light buttery yellow, slightly raised, with a more prominent whitish centre after $48 \mathrm{~h}$ of incubation. $L$. monocytogenes colonies are surrounded by a distinct zone of haemolysis. L. innocua shows no haemolytic zone; L. seeligeri produces zones of haemolysis that are less prominent than those of $L$. monocytogenes. The other Listeria species react in the same way as L. innocua (Beumer et al., 1997).

When possible, five typical colonies from the three plating media were streaked on Tryptone soy yeast extract agar (Tryptone soy broth (Lab M LAB4 $)+6 \mathrm{~g} / 1$ yeast extract $(\mathrm{Lab} \mathrm{M}, \mathrm{MC} 1)+12$ $\mathrm{g} / 1$ agar (Lab M, MC2) and incubated at $37^{\circ} \mathrm{C}$ for $24 \mathrm{~h}$.

The sample was considered positive for Listeria spp. if it was confirmed positive from at least one medium, and negative if it was confirmed negative from all the plating media.

\subsection{Confirmation and identification procedures}

All the isolates were confirmed to the genus level by Gram, catalase and oxidase tests, and tumbling motility (Tryptone soy broth, $25^{\circ} \mathrm{C}, 24 \mathrm{~h}$ ), and to the species level by API Listeria (BioMérieux, 10300) and by the Christie-Atkins-Munch-Peterson (CAMP) (Christie et al., 1944, cited by Farber and Peterkin, 1991) test with Staphylococcus aureus ATCC 25923 (Food Quality Centre, Escola Superior de Biotecnologia, Porto, Portugal) and Rhodococcus equi NCTC 1691 (Leatherhead Food Research Association, Surrey, UK) on sheep blood agar plates (BioMérieux, 43041).

Fifty-seven L. monocytogenes isolates were serotyped according to Seeliger and Hohne (1979) in the Food Safety Microbiology Laboratory in London in collaboration with Dr. James McLauchlin, using in-house produced anti-sera.

\section{Results and discussion}

A total of 201 fish and 42 environmental samples were analysed. L. monocytogenes was recovered from the fresh swordfish, fresh salmon-trout, vacuum packed cold-smoked salmon-trout, fresh salmon, vacuum packed cold-smoked salmon and from the water of the lake where the salmon trout are farmed. The overall frequency of Listeria spp. found in this experiment was $17 \%(42 / 243)$. L. monocytogenes, L. innocua, L. seeligeri, L. grayi and L. welshimeri were present respectively in $12,22,11,2$, and 1 samples. Twenty-one strains (37\%), isolated from in-processing fish were serovar $4 \mathrm{~b}$, while thirty-six strains $(63 \%)$, isolated from fresh salmon-trout, from the lake where the trout is farmed and from fresh salmon were serovar $1 / 2 \mathrm{a}$.

It is important to remark that the elective ability of EHA to differentiate L. monocytogenes and $L$. seeligeri from the other Listeria spp., must be accomplished by the guarantee that this medium is also a good alternative to traditional Oxford and PALCAM selective plating media, showing at least, equal capability for recovering Listeria spp. from food samples. For this purpose, colonies should be randomly picked up from the three plating media based respectively on esculin hydrolysis differentiation (Oxford and PALCAM) and appearance under UV and daylight based on the description of Beumer et al. (1997) (EHA). Of course it was impossible to ignore the differentiation among Listeria spp. in EHA based on the occurrence of haemolysis when picking up the presumptive positive Listeria colonies. This fact did not matter when just one species was 
noticeable in the plate. When more than one Listeria species were present on the EHA plate, the five Listeria spp. colonies, were picked up independently of showing or not haemolysis, to include all the different species present on the plate. For this last reason, in this study, it was possible to compare the ability of the three plating media to detect the genus Listeria but not to evaluate their ability for detecting each particular species. The ability of EHA for distinguishing L. monocytogenes from the other Listeria spp. colonies was also evaluated.

The recovery of Listeria spp. by the three plating media is shown in Table 1. The abilities of the three media to detect Listeria spp. varied depending on whether the samples were enriched in half-Fraser broth or in Fraser from 24 or $48 \mathrm{~h}$. All the media were shown to be less effective when isolating from Fraser primary enrichment where the competitive microbiota can inhibit or mask the Listeria colonies. Oxford medium was the best recovery medium from half-Fraser. EHA was the best recovery medium from Fraser broth after $24 \mathrm{~h}$ of incubation. Although, EHA detected one less positive sample than the other agars from Fraser after $48 \mathrm{~h}$, overall, the abilities of the three agars for recovering Listeria spp. were considered not markedly different. However, it should be remarked that only 34 of the 42 Listeria spp. confirmed samples (at least positive by one method) were simultaneously positive by all the plating media. This finding emphasises the importance of using more than one protocol for the detection of Listeria spp. The same conclusion was reported by Duarte et al. (1999), after comparing four different protocols for detection of Listeria spp. in the same type of products. Although L. monocytogenes was found in 34 samples after isolation from either Oxford or PALCAM agars, only five samples were simultaneously positive in all the protocols. If the isolation on either of the selective agars was considered, only nine samples would be simultaneously positive for L. monocytogenes in all four broths tested. These differences between methods underline the probability of underestimating the occurrence of L. monocytogenes when just one protocol is used for its detection.

The species composition of the 42 Listeria spp.positive samples is shown in Table 2. On EHA, most of L. monocytogenes colonies were easily distinguished from the other Listeria spp. colonies. The $L$. monocytogenes colonies were surrounded by a distinct zone of haemolysis, whereas $L$. innocua and $L$. grayi showed no haemolysis and $L$. seeligeri showed a very faint zone of haemolysis. L. welshimeri was only recovered from PALCAM agar. As mentioned above, purposefully not only $L$. monocytogenes typical colonies but also the other Listeria spp. typical colonies were picked up from EHA to assure the diversity of the species recovered, and thus to omit the differentiation capability at species level, just highlighting its selectivity behaviour at the genus level. If only presumptive positive $L$. monocytogenes colonies were picked up from EHA, it would be impossible to observe the similarity of species composition in the positive samples detected by the three selective agars (Table 2).

For evaluation of the most important feature of EHA - the elective ability of distinguishing $L$. monocytogenes from the other Listeria spp. colonies

Table 1

Comparison of Oxford, PALCAM and EHA selective plating media for isolation of Listeria spp.

\begin{tabular}{lllllllll}
\hline Enrichment broth & Oxford & PALCAM & EHA & Only Oxford $^{\text {a }}$ & Only PALCAM $^{\text {b }}$ & Only EHA $^{\mathrm{c}}$ & Three media $^{\mathrm{d}}$ & Either medium $^{\mathrm{e}}$ \\
\hline Half-Fraser & $34 / 38$ & $31 / 38$ & $32 / 38$ & $3 / 38$ & $0 / 38$ & $3 / 38$ & $27 / 38$ & $38 / 42$ \\
Fraser, 24 h & $36 / 42$ & $38 / 42$ & $40 / 42$ & $1 / 42$ & $0 / 42$ & $3 / 42$ & $34 / 42$ & $42 / 42$ \\
Fraser, 48 h & $38 / 42$ & $38 / 42$ & $37 / 42$ & $1 / 42$ & $1 / 42$ & $2 / 42$ & $33 / 42$ & $42 / 42$ \\
Total positives & $40 / 42$ & $40 / 42$ & $41 / 42$ & $0 / 42$ & $0 / 42$ & $2 / 42$ & $23 / 42$ & 42 \\
\hline
\end{tabular}

\footnotetext{
${ }^{\mathrm{a}}$ Listeria spp.-positive samples detected only by Oxford agar.

${ }^{\mathrm{b}}$ Listeria spp.-positive samples detected only by PALCAM agar.

${ }^{\mathrm{c}}$ Listeria spp.-positive samples detected only by EH agar.

${ }^{\mathrm{d}}$ Listeria spp.-positive samples detected by the three media simultaneously.

${ }^{\mathrm{e}}$ Listeria spp.-positive samples detected by any of the three media.
} 
Table 2

Species composition of the 42 Listeria spp.-positive samples

\begin{tabular}{|c|c|c|c|c|c|c|c|}
\hline Medium & Enrichment broth & L. monocytogenes & L. innocua & L. seeligeri & L. welshimeri & L. grayi & Total \\
\hline \multirow[t]{3}{*}{ Oxford } & Half-Fraser & 9 & 17 & 8 & 0 & 0 & $34 / 42$ \\
\hline & Fraser, $24 \mathrm{~h}$ & 10 & 20 & 8 & 0 & 0 & $38 / 42$ \\
\hline & Fraser, $48 \mathrm{~h}$ & 9 & 20 & 9 & 0 & 0 & $38 / 42$ \\
\hline \multirow[t]{3}{*}{ PALCAM } & Half-Fraser & 8 & 16 & 7 & 0 & 0 & $31 / 42$ \\
\hline & Fraser, $24 \mathrm{~h}$ & 9 & 18 & 10 & 1 & 1 & $39 / 42$ \\
\hline & Fraser, $48 \mathrm{~h}$ & 10 & 20 & 8 & 0 & 0 & $38 / 42$ \\
\hline \multirow[t]{3}{*}{ EHA } & Half-Fraser & 8 & 17 & 7 & 0 & 0 & $32 / 42$ \\
\hline & Fraser, $24 \mathrm{~h}$ & 8 & 22 & 10 & 0 & 0 & $40 / 42$ \\
\hline & Fraser, $48 \mathrm{~h}$ & 9 & 19 & 9 & 0 & 1 & $38 / 42$ \\
\hline
\end{tabular}

based on the haemolytic activity-33 suspect $L$. monocytogenes colonies (presumptive positive $L$. monocytogenes (P.P.)) and 79 suspect Listeria spp. colonies (presumptive negative L. monocytogenes (P.N.)) from EHA plates were identified by the procedures described above. These results are shown in Table 3. EHA identified correctly 76 Listeria spp.

Table 3

Ability of EHA to distinguish L. monocytogenes colonies from the colonies of other Listeria spp. based on haemolytic properties

\begin{tabular}{|c|c|c|}
\hline $\begin{array}{l}\text { Enrichment } \\
\text { broth }\end{array}$ & $\begin{array}{l}\text { Confirmed as } \\
\text { correct (C.C.) }\end{array}$ & $\begin{array}{l}\text { Confirmed as } \\
\text { incorrect (C.I.) }\end{array}$ \\
\hline \multicolumn{3}{|l|}{ Half-Fraser } \\
\hline P.P. (14) & $9(\mathrm{a})$ & $5(\mathrm{~b})$ \\
\hline P.N. (20) & $20(c)$ & $0(d)$ \\
\hline \multicolumn{3}{|l|}{ Fraser, $24 h$} \\
\hline P.P. (10) & $6(a)$ & $4(\mathrm{~b})$ \\
\hline P.N. (30) & $28(\mathrm{c})$ & $2(d)$ \\
\hline \multicolumn{3}{|l|}{ Fraser, $48 h$} \\
\hline P.P. (9) & $8(a)$ & $1(\mathrm{~b})$ \\
\hline P.N. (29) & $28(\mathrm{c})$ & $1(\mathrm{~d})$ \\
\hline \multicolumn{3}{|l|}{ Total } \\
\hline P.P. (33) & 23 (a) & $10(b)$ \\
\hline P.N. (79) & $76(\mathrm{c})$ & $3(\mathrm{~d})$ \\
\hline
\end{tabular}

P.P.: presumptive positive (showing haemolysis); P.N.: presumptive negative.

a: Correctly identified presumptive positive and confirmed as L. monocytogenes.

b: Presumptive positive L. monocytogenes colonies confirmed to be other Listeria spp.

c: Correctly identified presumptive negative and confirmed to be other Listeria spp.

d: Presumptive negative results confirmed to be positive for $L$. monocytogenes. and presented only three false-negative results ( $L$. monocytogenes colonies showing no noticeable haemolysis on EHA but CAMP-positive). Twentythree of the thirty-three L. monocytogenes presumptive positive colonies, were confirmed to be positive and ten were confirmed to be L. seeligeri.

The three false-negative colonies were never recovered from half-Fraser broth, whereas 5 of the 10 false-positive colonies were recovered from this primary enrichment. The major problem of distinguishing L. monocytogenes colonies from $L$. seeligeri colonies in plates of Fraser primary enrichment, was due to the greater growth of competitive microbiota, and consequently increasing difficulty of differentiating the corresponding typical haemolysis zone. From Fraser after $48 \mathrm{~h}$, only one L. seeligeri was misidentified as L. monocytogenes.

The presence of acriflavine might lead to decreased recovery of $L$. monocytogenes from EHA (Beumer et al., 1997). As this selective agent is known to repress haemolysis, $L$. monocytogenes colonies might be misidentified as other Listeria spp. However, studies with L. monocytogenes blood agar (LMBA), another Listeria spp. selective plating medium based on haemolytic differentiation where the selectivity of the medium was reduced compared to EHA (no acriflavine), stated that a slightly higher selectivity would have been required for cold-smoked fish samples with high levels of competitive bacteria (Johansson, 1998).

The results of the present study confirm the elective ability of EHA to distinguish L. monocytogenes from the other Listeria spp., a finding that can improve the detection of the pathogen earlier than by using the standard media Oxford and PALCAM. As 
reported by Beumer et al. (1997) and referred previously, the selective plating media used in traditional cultural procedures are not designed to differentiate Listeria spp. and the selection of five suspect colonies at random from such media could lead to the detection only of Listeria spp. other than L. monocytogenes, even though the latter was present on the plate. However, as the ability of EHA to distinguish Listeria spp. is based on the occurrence of haemolysis, false-negatives (due to non-haemolytic L. monocytogenes strains) and false-positives (due to the presence of L. seeligeri) might occur (Beumer et al., 1996). In this study, no non-haemolytic L. monocytogenes were found but a few non-haemolytic $L$. monocytogenes strains were found in the same smokeries in a previous survey (Duarte et al., 1995). As in this case where EHA cannot differentiate non-haemolytic Listeria spp. from L. monocytogenes colonies, further additional tests are required. Thus, colonies isolated by culturing methods must always be confirmed even when EHA is used.

However, despite the advantage of using this selective differential isolation medium as a rapid screening alternative to time-consuming classical identification, unless it is produced as a commercial medium, the preparation is both time- and labourconsuming.

Furthermore, as referred previously, rather than promoting a single method for isolation of Listeria spp., the benefit of using a combination of methods should be stressed.

\section{Acknowledgements}

The authors gratefully acknowledge EU FAIR Project CT95-1207 "Spoilage and Safety of ColdSmoked Fish" for financial support.

\section{References}

Autio, T., Hielm, S., Miettinen, M., Sjöberg, A.-M., Aarnisalo, K., Björkroth, J., Mattila-Sandholm, T., Korkeala, H., 1999. Sources of Listeria monocytogenes contamination in a coldsmoked rainbow trout processing plant detected by pulsed-field gel electrophoresis typing. Appl. Environ. Microbiol. 65, 150155 .
Ben Embarek, P., 1994. Presence, detection and growth of Listeria monocytogenes in seafoods: a review. Int. J. Food Microbiol. 23, 17-34.

Beumer, R.R., te Giffel, M.C., Kok, M.T.C., Rombouts, F.M., 1996. Confirmation and identification of Listeria spp. Lett. Appl. Microbiol. 22, 448-452.

Beumer, R.R., te Giffel, M.C., Cox, L.J., 1997. Optimization of enhanced haemolysis agar (EHA), a selective medium for the isolation of Listeria monocytogenes. Listeria monocytogenes Detection and Behaviour in Food and in the Environment (Thesis Landbouwuniversiteit Wageningen). Koninklijke Bibliotheek, Den Haag, Netherlands, pp. 55-68.

Dillon, R., Patel, T., Ratnam, S., 1992. Prevalence of Listeria in smoked fish. J. Food Prot. 55, 866-870.

Duarte, G., Vaz-Velho, M., Gibbs, P., 1995. Occurrence of Listeria spp. and Listeria monocytogenes in Portuguese coldsmoked fish products. Arq. INSA 20/21, 55-61.

Duarte, G., Vaz-Velho, M., Capell, C., Gibbs, P., 1999. Comparison of the efficacy of four individual selective enrichment protocols and their combination for isolating Listeria spp. from environmental and fish samples along their cold-smoking processing chains. Int. J. Food Microbiol. 52, 163-168.

Eklund, M.W., Poysky, F.T., Paranjpye, R.N., Lashbrook, L.C., Peterson, M.E., Pelroy, G.A., 1995. Incidence and sources of Listeria monocytogenes in cold-smoked fishery products and processing plants. J. Food Prot. 58, 502-508.

Ericsson, I.L., Eklow, A., Danielsson-Tham, M.L., Loncarevic, S., Mentzing, O., Persson, I., Unnerstad, H., Tham, W., 1997. An outbreak of listeriosis suspected to have been caused by rainbow trout. J. Clin. Microbiol. 35, 2904-2907.

Farber, J., 1991. Listeria monocytogenes in fish products. J. Food Prot. 54, 922-924, 934.

Farber, J.M., Peterkin, P.I., 1991. Listeria monocytogenes, a foodborne pathogen. Microbiol. Rev. 5, 476-511.

Fuchs, R.S., Nicolaides, L., 1994. Incidence of Listeria in hot and cold-smoked fish. Lett. Appl. Microbiol. 19, 394-396.

Gibson, D., 1992. Pathogenic microorganisms of importance in seafood. In: Huss, H., Jacobsen, H., Liston, M. (Eds.), Quality Assurance in the Fish Industry. Elsevier, Amsterdam, pp. 197-209.

Hartemink, R., Georgsson, F., 1991. Incidence of Listeria species in seafood and seafood salads. Int. J. Food Microbiol. 12, 189-196.

Hudson, J.A., Mott, S.J., Delacy, K.M., Edridge, A.L., 1992. Incidence and coincidence of Listeria spp., motile aeromonads and Yersinia enterocolitica on ready-to-eat fleshfoods. Int. J. Food Microbiol. 16, 99-108.

International Standard ISO 11290-1 1996. Technical Committee ISO/TC 34, Agricultural food products, Subcommitee SC 9, Microbiology. Geneve, Switzerland.

Jemmi, T., Keusch, A., 1994. Occurrence of Listeria monocytogenes in freshwater fish farms and fish-smoking plants. Food Microbiol. 11, 309-316.

Johansson, T., 1998. Enhanced detection and enumeration of Listeria monocytogenes from foodstuffs and food-processing environments. Int. J. Food Microbiol. 40, 77-85.

Jørgensen, L.V., Huss, H.H., 1998. Prevalence and growth of 
Listeria monocytogenes in naturally contaminated seafood. Int. J. Food Microbiol. 42, 127-131.

Loncarevic, S., Tham, S., Danielsson-Tham, M.-L., 1996. Prevalence of Listeria monocytogenes and other Listeria spp. in smoked and "Gravad" fish. Acta Vet. Scand. 37, 13-18.

Lovett, J., Twedt, R., 1988. Listeria. Outstanding symposia in food science and technology. Food Technol. 188-191 (April).

MacDonald, F., Sutherland, A., 1994. Important differences between the generation times of Listeria monocytogenes and $L$. innocua in two Listeria enrichment broths. J. Dairy Res. 61, 433-436.

Rørvik, L.M., Caugant, D.A., Yndestad, M., 1995. Contamination pattern of Listeria monocytogenes and other Listeria spp. in a salmon slaughterhouse and smoked salmon processing plant. Int. J. Food Microbiol. 25, 19-27.

Seeliger, H.T.R., Hohne, K.P., 1979. Serotyping of Listeria momocytogenes and selected species. In: Bergen, T., Norris, J.T. (Eds.), Methods in Microbiology, vol. 13, Academic Press, London, pp. 31-49.

Vaz-Velho, M., Duarte, G., Gibbs, P., 1998. Occurrence of Listeria spp. in salmon-trout (Oncorhynchus mykiss) and salmon (Salmo salar). Food Sci. Technol. Int. 4, 121-125.

Vaz-Velho, M., Duarte, G., Gibbs, P., 2001. Comparison of peptone water and Fraser base, as pre-enrichment broths for recovering Listeria spp. from salmon and salmon-trout. Food Control (in press). 